\title{
Islet Transplantation for Type 1 Diabetes
}

\author{
BORIS GALA-LOPEZ*1), TOSHIYASU KAWAHARA*2), \\ ANDREW R. PEPPER*11, A.M. JAMES SHAPIRO*1) \\ *1) Clinical Islet Transplant Program. Department of Surgery, University of Alberta, Alberta, Canada, *2) Division of \\ Gastroenterological and Transplant Surgery, Department of Surgery, Asahikawa Medical University, Hokkaido, Japan \\ Decades of research have brought islet transplantation from the dream of few to the reality of many. This \\ procedure started as an experimental method to treat type 1 diabetes mellitus, which features $\beta$-cell dysfunction \\ due to auto immunity. The burden of complication in these patients is cumulative and has become a major health \\ problem, despite the availability of insulin therapy. The procedure relies on a sequence of finely orchestrated \\ procedures starting in the donor and followed by several steps to isolate high quality islets. Extensive research is \\ nowadays focussed in improving islet engraftment by providing a more beneficial environment to newly \\ transplanted cells, coupled by more effective immunosuppressive drugs to avoid allo and auto immunity. Excellent \\ results are now a reality in the most specialized centers. Yet, further steps are required to transform this low-risk \\ treatment in a widely available and long-lasting therapy for diabetics worldwide.
}

Key words: islet transplantation, diabetes mellitus, graft survival

\section{Introduction}

Type 1 diabetes Mellitus (T1DM) is a chronic, autoimmune disease resulting from the destruction of the insulin-producing $\beta$-cells within the pancreatic islets. This disease is characterized by impaired glucose metabolism leading to chronic micro and macrovascular complications ${ }^{12}{ }^{2)}$. Despite ongoing advances in monitoring and treatment of diabetes, morbidity and mortality remains increased when compared with non-diabetic populations ${ }^{12}{ }^{2}$.

Current treatment for T1DM mainly relies on the use of insulin replacement to attain normoglycemia. However, the metabolic control resulting from insulin therapy may not be accurate and is not sufficient to prevent long-term complications ${ }^{3)}$. Transplants have therefore become a valid alternative for these patients given the current results and despite the risk associated with the procedure and the long-term immunosuppression ${ }^{4)}$ ).
Significant progress has occurred in the outcomes of clinical islet transplantation, due to significant development in immunosuppression and availability of high quality of islets preparations for transplantation. Since the introduction of the Edmonton Protocol, when sustained c-peptide production and successful insulin independence was reported after solitary islet transplantation, this procedure has become an accepted modality to stabilize frequent hypoglycemias or severe glycemic lability in highly selected subjects with poor diabetic control ${ }^{6)-8}$. This new breakthrough was possible with the avoidance of corticosteroids, and high-quality islet preparations. This mini-review presents the important role of islet transplantation, some of the challenges it faces and potential improvements for the future.

Corresponding author: Boris Gala-Lopez

Immunobiology \& Islet Transplantation Research, Clinical Islet Transplant Program, Alberta Diabetes Institute, University of Alberta 5-040 Li Ka Shing Health Center for Research Innovation

116 St \& 85 Avenue. Edmonton, AB. Canada T6G 2R3

TEL: +1-780-492-4656 (Lab) FAX: + 1-780-492-2892

Special Reviews: Minimally Invasive Hepatobiliary and Pancreatic Surgery

〔Received Jan. 7, 2015〕 


\section{Historical perspective}

Clinical islet transplant was first attempted in the XIX century when early and rudimentary experimental treatments were performed by Dr. WatsonWilliams and colleagues, consisting of subcutaneous implantation of a sheep's pancreas ${ }^{9)}{ }^{10)}$. Several years later (1960) the vision changed to isolating cells rather than transplanting pancreas fragments. Paul E. Lacy pioneered experiments in mice, later refined by mechanical enzymatic digestion and by the use of dialyzed Ficoll for more efficient islet separation $\left.{ }^{9)}{ }^{11}\right)_{12}$.

Various research groups were working in improving techniques that allow a larger yield and the intraductal injection of collagenase proved to be the most effective method for the successful isolation of islets from large animals, including humans ${ }^{13)}$. The introduction of a semi-automated dissociation chamber originally developed by Ricordi et al. in 1988 was definitely a major revolution in the process of obtaining high quality cells ${ }^{14)}$. The "Ricordi Cham-

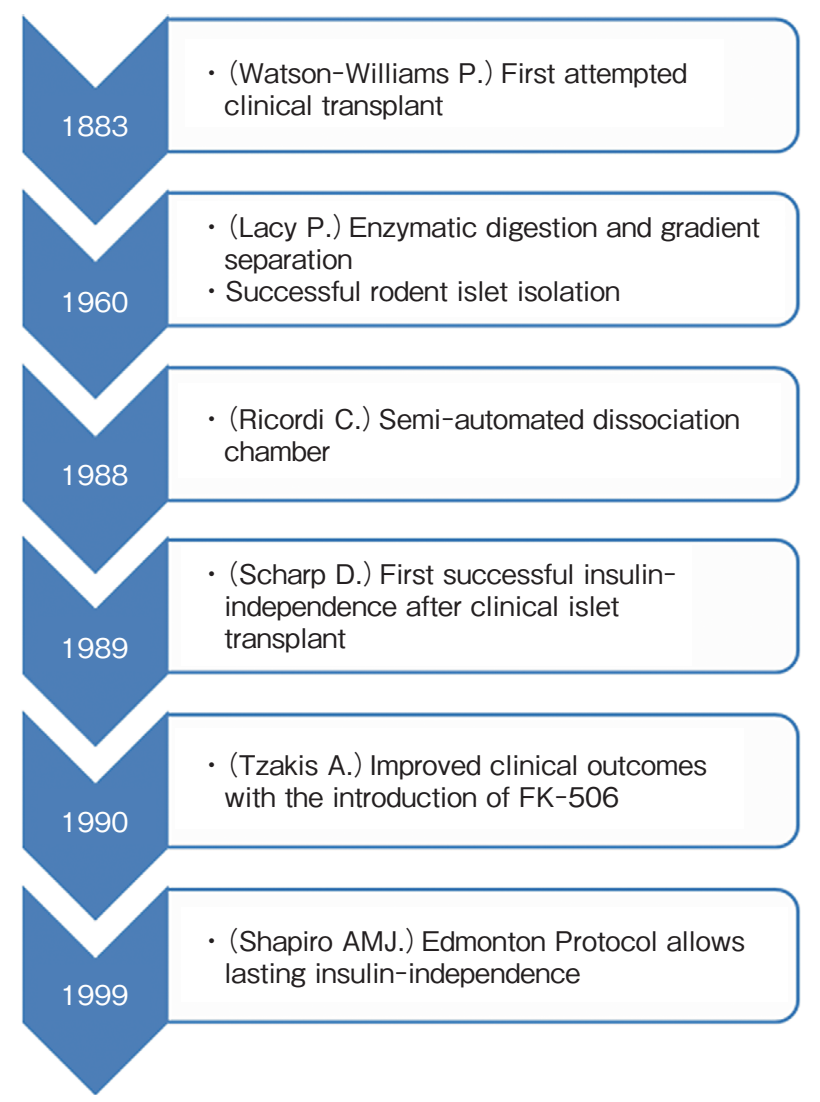

Figure-1 Timeline with the most important milestones in the transition of islet transplantation from experimental to clinical therapy ber" along with the COBE 2991 refrigerated centrifuge and a highly purified enzyme blend are today key elements to consistently achieve clinically relevant islet yield, with improved viability and function ${ }^{9}$.

These studies allowed the establishment of successful transplantation in diabetic rodents and paved the way to eventually allow reversal of diabetes in the first human patient in 1989, followed by significant improvement when enhanced immunosuppression was introduced ${ }^{15)-17}$.

The Edmonton Protocol was definitely a milestone in the ultimate implementation of previous findings into a more efficient method to achieve lasting normoglycemia in a human cohort. Shapiro AMJ and collaborators combined the use of sufficient islet mass $(\sim 11,500$ islet equivalent $/ \mathrm{kg})$ with a steroid-free immunosuppressive regime. Induction was achieved with daclizumab, followed by a combination of sirolimus and low-dose tacrolimus ${ }^{6)}$. This new protocol resulted in $100 \%$ insulin independence at 1 year and was rapidly implemented in all existing transplant centers.

Figure- 1 summarizes the most important milestones from this initial era in the development of islet transplantation from experimental to clinical therapy.

\section{The process of islet transplantation}

The process to obtain high quality islets for transplantation starts with the adequate selection of donors. Previous studies have identified various donor-related variables affecting islet isolation outcome; including donor age, cause of death, body mass index (BMI), cold ischemia time, length of hospitalization, use of vasopressors, and blood glucose levels ${ }^{18)}{ }^{19)}$.

Preserved pancreases are then put through the digestion process via injection of an enzyme preparation to distend the main duct and elicit tissue separation after mechanical disruption ${ }^{20)}$. The Ricordi Chamber is crucial for this step, which requires a precise temperature control to allow proper results ${ }^{14)}$. High purity islet fractions are then obtained after using Ficoll gradients, being this density dependent separation of islets from exocrine tissue the most simple and effective approach for islet purification ${ }^{12)}{ }^{20}$. The methodology is based on the principle that, during centrifugation, tissue 
will migrate and settle to the density that is equal to its own density ${ }^{20)}$. The use of COBE 2991 refrigerated centrifuge has largely revolutionized this process, allowing highly improved yields. The final islet preparation is kept in culture until an adequate HLA-matched recipient is identified.

The transplant procedure has evolved in time to allow current results. Today the intraportal access has become gold standard with minimal risk for the patients and the best engraftment possibility for diabetic patients ${ }^{21)}$. However, despite the latest refinements in the procedure and enhanced immunosuppression, islet must overcome significant obstacles to engraft, survive and function for a long time.

\section{Sites for transplantation}

Success in islet engraftment is very much dependent on the availability of abundant vessels to allow a proper exchange between islets and the surrounding tissues ${ }^{22}$. Multiple sites for transplantation have been studied with clear differences in performance and efficiency. These sites include the intraportal site, the kidney subcapsular space, the splenic pulp, the omentun, testes, epididimal fat, the eye chamber, gastric submucosa, the subcutaneous space, vein sacs and small bowel, among others ${ }^{22)}$.

Intraportal infusion has been recognized as the most clinically efficient site for implantation given its high vascularity, the proximity to nutrient factors, and the physiological first pass insulin delivery to the liver ${ }^{9}$. However, this site also carries potential risk for the recipient, including post-procedural haemorrhage or thrombosis, acute portal hypertension and arteriovenous fistula ${ }^{211}$.

\section{The main obstacles}

Islet transplantation through the portal vein is minimally invasive but results in islet entrapment within the sinusoids. This space provides an opportunity to acquire oxygen and nutritional support. However, the appropriate vascular connections are only formed around two weeks after infusion, leaving a large portion of the graft on ischemic conditions ${ }^{22)}$. The presence of tissue factor associated with the instant blood-mediated inflammatory reaction (IBMIR) and subsequent platelet activation, clot formation, and lymphocyte recruitment, may also negatively influence initial islet survival and overall transplant outcome ${ }^{23)}{ }^{24}$.

There are immunological challenges to islet survival too. Allo-immune rejection is a very important factor characterized by a full-blown response upon transplantation. However, autoimmune destruction is also present due to the inner nature of the disease featuring $\beta$-cell autoantibodies and $\beta$-cell-specific autoreactive T-cells. This type of response demands biological strategies to overcome immune response and eventually elicit tolerance in the host ${ }^{25}$. The agents used vary in nature and are permanently needed after transplantation.

This continuous exposure to immunosuppressants may have an adverse impact on islet function and revascularization ${ }^{26}$. Tacrolimus and sirolimus are two well-known and widely used drugs in the settings of transplant in general and also in islet transplantation, with proven deleterious (diabetogenic) effects on $\beta$-cell mass over time ${ }^{27)-30)}$.

\section{Graft function monitoring}

The overall results of clinical islet transplantation have significantly improved over the years reaching up to $50 \%$ graft survival after 5 years for selected centers. Yet, the profile of $\beta$-cell function of these individuals does not compare to healthy counterparts ${ }^{4)}$ 31). Multiple factors still account for the loss of islet mass, including immunological events, islet exhaustion and drug cytotoxicity. Thus, continuous monitoring of the graft function is paramount to secure long-term success.

The main tools to positively confirm islet health are relatively frequent clinical indicators. Levels of blood glucose, stimulated C-peptide, Hb1AC and insulin requirements are among the parameters evaluated in recipients. These variables may be analyzed individually or combined as scoring systems such as: the Beta Score, the Mean Amplitude Glycemic Excursions (MAGE), Lability Index, Hypo Score, Arginine Stimulation Score, the SUITO Index, etc. They all provide objective and quantitative information about the graft function and serve as important decision-making tools for the clini$\operatorname{cian}^{9)}{ }^{32-34)}$.

Complementary imaging studies may also be used to visualize the islets, with a high value when 
correlated with scoring systems. Techniques like optical imaging, magnetic resonance imaging and positron emission tomography are currently evaluated experimentally and have demonstrated potential for clinical application in the near future ${ }^{35)}$ 36).

\section{Future directions for islet transplantation}

Islet transplant has evolved in time and today is performed in various centers with long-term results comparable to whole organ transplant. However, a significant number of patient undergoing this procedure still require multiple infusions to achieve normoglycemia and at some point some of them return to insulin supplementation. Yet, being insulin-dependent again does not necessarily equate with complete loss of graft function, as many of these patients remain C-peptide positive.

Several pre-clinical and clinical projects are now focused in finding improved results in islet transplantation by introducing tolerance-inducing medi-
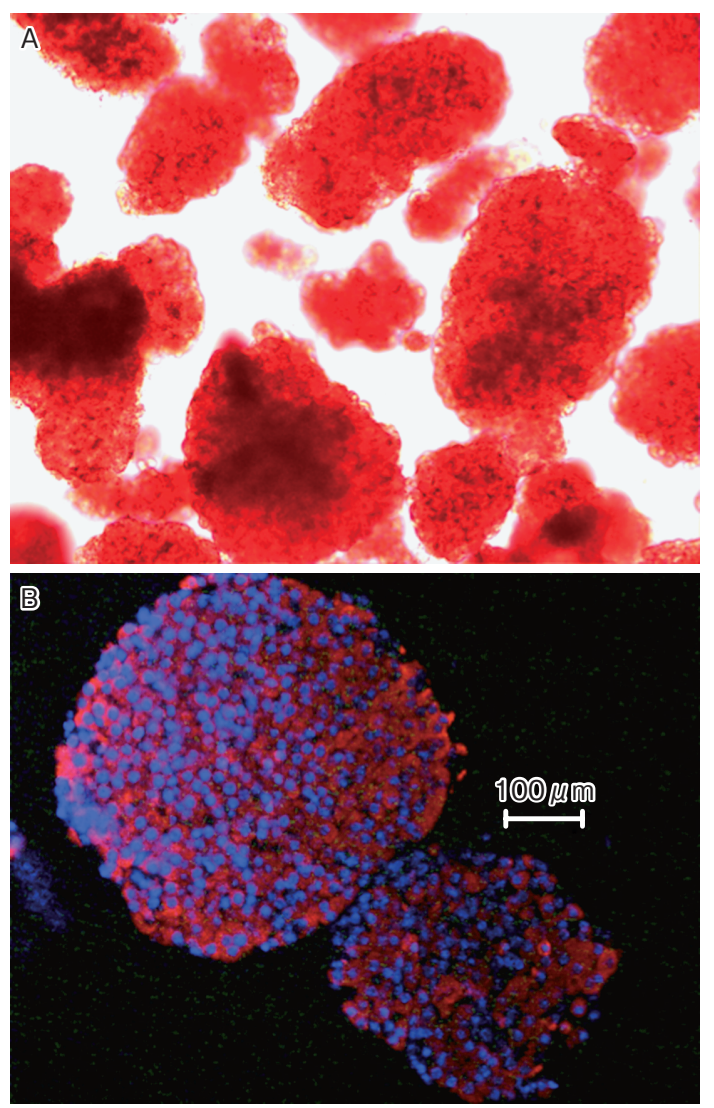

Figure-2 Isolated mouse islets

A. Islets stained with dithizone (DTZ, Sigma) under bright field microscopy (20X).

B. Fluorescent microscopy of islets dually stained for insulin (red) and nuclei (DAPI, blue) (40X). cation. New potent biological agents like humanized anti -CD154, anti -CD28 or anti -CD52, among others. These drugs have shown very encouraging results in solid organ transplant and are expected to produce enhanced islet survival after transplant ${ }^{25}$. Figure-2 shows isolated mouse islets, which are a basic tool in transplant experimentation.

Another stream of investigation is $\mathrm{co}^{-}$-transplantation of islets with cells providing immune-protection and/or anti-inflammatory effects; such as Sertoli cells and mesenchymal stem cells. An important alternative is the use of surrogate insulin-producing stem cells or xenotransplantation as a source of unlimited supply of islets ${ }^{4)}$.

\section{Conclusion}

Islet transplantation is a treatment option for patients with type I diabetes, allowing glucose homeostasis without exogenous insulin administration. The procedure is very safe and currently provides extensive protection from hypoglycaemic episodes for as much as 5 years. However, approximately $60 \%$ of the graft is lost soon after infusion due to various mechanisms. Preventing this significant post-transplant cell death is strategic and would have an immediate impact on the procedure.

Opportunities for intervention are endless to diminish the damage associated with the isolation, preservation, engraftment and immune-protection. These strategies would reduce the amount of islets required per patient to attain normoglycemia and would open up the avenue for using surrogate cells in clinical practice.

Preventing allo and auto-immunity in islet transplantation demands lifelong immunosuppression. Many of the new developments in the field are devoted to immunoprotection and to induction of tolerance with new and more potent biological agents. Other technologies, like the use of bio-engineered devices or encapsulation are also used looking to avoid the need for immunosuppression altogether.

Evidences show that success may come from the combination of different approaches to achieve a synergistic solution to the current limitations of islet transplantation. 


\section{Conflict of Interest}

The author has no conflict of interest with this manuscript.

\section{References}

1) Forbes JM, Cooper ME: Mechanisms of diabetic complications. Physiol Rev, 2013; 93: 137-188.

2) Vazeou A: Continuous blood glucose monitoring in diabetes treatment. Diabetes Res Clin Pract, 2011; 93 Suppl 1: S125-130.

3) Merani S, Shapiro AM: Current status of pancreatic islet transplantation. Clin Sci (Lond), 2006; 110: 611-625.

4) Pepper AR, Gala-Lopez B, Ziff O, Shapiro AJ: Current status of clinical islet transplantation. World J Transplant, 2013; 3: 48-53.

5) Chhabra P, Brayman KL: Current status of immunomodulatory and cellular therapies in preclinical and clinical islet transplantation. J Transplant, 2011; 2011: 637692.

6) Shapiro AM, Lakey JR, Ryan EA, et al: Islet transplantation in seven patients with type 1 diabetes mellitus using a glucocorticoid-free immunosuppressive regimen. N Engl J Med, 2000; 343: 230-238.

7) Shapiro AM, Ricordi C, Hering BJ, et al: International trial of the Edmonton protocol for islet transplantation. N Engl J Med, 2006; 355: 1318-1330.

8) Ryan EA, Paty BW, Senior PA, et al: Five-year followup after clinical islet transplantation. Diabetes, 2005; 54: 2060-2069.

9) Shapiro AMJ: A historical perspective on experimental and clinical islet transplantation. In: Shapiro AMJ, Shaw JAM, eds. Islet Transplantation and Beta Cell Replacement Therapy. New York: Informa Healthcare, 2007.

10) Watson-Williams P: Notes on diabetes treated with extract and by grafts of sheep's pancreas. Br Med J, 1894; 2: 1303-1304.

11) Lacy PE, Kostianovsky M: Method for the isolation of intact islets of Langerhans from the rat pancreas. Diabetes, 1967; 16: 35-39.

12) Scharp DW, Kemp CB, Knight MJ, Ballinger WF, Lacy P: The use of Ficoll in the preparation of viable islet of Langerhans from the rat pancreas. Transplantation, 1973; 16: 686-689.

13) Lakey JR, Warnock GL, Shapiro AM, et al: Intraductal collagenase delivery into the human pancreas using syringe loading or controlled perfusion. Cell Transplant, 1999; 8: 285-292.

14) Ricordi C, Lacy PE, Scharp DW: Automated islet isolation from human pancreas. Diabetes, 1989; 38 Suppl 1: 140-142.

15) Scharp DW, Lacy PE, Santiago JV, et al: Insulin independence after islet transplantation into type I diabetic patient. Diabetes, 1990; 39: 515-518.

16) Tzakis AG, Ricordi C, Alejandro R, et al: Pancreatic islet transplantation after upper abdominal exenteration and liver replacement. Lancet, 1990; 336: 402-405.

17) Ricordi C, Tzakis AG, Carroll PB, et al: Human islet isolation and allotransplantation in 22 consecutive cases. Transplantation, 1992; 53: 407-414.

18) Kin T, Murdoch TB, Shapiro AM, Lakey JR: Estimation of pancreas weight from donor variables. Cell Trans- plant, 2006; 15: 181-185.

19) O'Gorman D, Kin T, Murdoch T, et al: The standardization of pancreatic donors for islet isolation. Transplant Proc, 2005; 37: 1309-1310.

20) Pepper AR, Gala-Lopez B, Kin T: Advances in clinical islet isolation. In: Islam MS, ed. Islets of Langerhans, 2nd ed. Dordrecht: Springer, 2014: 1165-1187.

21) Kawahara T, Kin T, Kashkoush S, et al: Portal vein thrombosis is a potentially preventable complication in clinical islet transplantation. Am J Transplant, 2011; 11: 2700-2707.

22) Pepper AR, Gala-Lopez B, Ziff O, Shapiro AM: Revascularization of transplanted pancreatic islets and role of the transplantation site. Clin Dev Immunol, 2013; 2013: 352315.

23) Hawthorne WJ, Salvaris EJ, Phillips P, et al: Control of IBMIR in neonatal porcine islet xenotransplantation in baboons. Am J Transplant, 2014; 14: 1300-1309.

24) Hwang JW, Jung HS, Lee DY: Inhibition of platelet adhesion onto intrahepatically transplanted islets using PEGylation for attenuating instant blood-mediated inflammatory reaction (IBMIR). J Control Release, 2011; 152 Suppl 1: e213-214.

25) Gala-Lopez B, Pepper AR, Shapiro AM: Biologic agents in islet transplantation. Curr Diab Rep, 2013; 13: 713-722.

26) Nishimura R, Nishioka S, Fujisawa I, et al: Tacrolimus inhibits the revascularization of isolated pancreatic islets. PLoS One, 2013; 8: e56799.

27) Hernandez-Fisac I, Pizarro-Delgado J, Calle C, et al: Tacrolimus-induced diabetes in rats courses with suppressed insulin gene expression in pancreatic islets. Am J Transplant, 2007; 7: 2455-2462.

28) Johnson JD, Ao Z, Ao P, et al: Different effects of FK506, rapamycin, and mycophenolate mofetil on glucosestimulated insulin release and apoptosis in human islets. Cell Transplant, 2009; 18: 833-845.

29) Li F, Li Q: Effects of different dose of FK506 on endocrine function of pancreatic islets and damage of beta cells of pancreatic islets in a Wistar rat model. Immunopharmacol Immunotoxicol, 2010; 32: 333-338.

30) Rostambeigi N, Lanza IR, Dzeja PP, et al: Unique cellular and mitochondrial defects mediate FK506-induced islet beta-cell dysfunction. Transplantation, 2011; 91: 615-623.

31) McCall M, Shapiro AM: Update on islet transplantation. Cold Spring Harb Perspect Med, 2012; 2: a007823.

32) Caumo A, Maffi P, Nano R, et al: Comparative evaluation of simple indices of graft function after islet transplantation. Transplantation, 2011; 92: 815-821.

33) Ryan EA, Shandro T, Green K, et al: Assessment of the severity of hypoglycemia and glycemic lability in type 1 diabetic subjects undergoing islet transplantation. Diabetes, 2004; 53: 955-962.

34) Matsumoto S, Noguchi H, Hatanaka N, et al: Evaluation of engraftment after single islet transplantation from a brain-dead donor by the secretory unit of islet transplant objects (SUITO) index. Transplant Proc, 2008; 40: 364-366.

35) Borot S, Crowe LA, Toso C, et al: Noninvasive imaging techniques in islet transplantation. Curr Diab Rep, 2011; 11: $375-383$

36) Speier S, Nyqvist D, Cabrera O, et al: Noninvasive in vivo imaging of pancreatic islet cell biology. Nat Med, 2008; 14: $574-578$. 